\title{
The downregulation of miR-125a-5p functions as a tumor suppressor by directly targeting MMP-11 in osteosarcoma
}

\author{
NIYAZI WARESIJIANG* , JUNGANG SUN*, REWUTI ABUDUAINI, \\ TAYIER JIANG, WENZHENG ZHOU and HONG YUAN
}

Department of Orthopaedics, People's Hospital of Xinjiang Uygur Autonomous Region, Urumqi, Xinjiang 830001, P.R. China

Received June 11, 2015; Accepted March 30, 2016

DOI: $10.3892 / \mathrm{mmr} .2016 .5141$

\begin{abstract}
Osteosarcoma is one of the most common primary malignant bone cancers in juveniles and adults. Increasingly, reports indicate that microRNAs (miRNAs) may provide novel therapeutic targets for cancer treatment. The aim of the present study was to investigate the expression of miR-125a-5p and to identify its functional significance in osteosarcoma. This indicated that miR-125a-5p was downregulated in osteosarcoma tissue and cell lines using reverse transcription-quantitative polymerase chain reaction. Following transfection with miR-125a-5p mimics or the negative control, cell migration, invasion and epithelial-mesenchymal transition (EMT) assays were conducted in osteosarcoma cells. These results indicated that the overexpression of $\mathrm{miR}-125 \mathrm{a}-5 \mathrm{p}$ resulted in inhibited osteosarcoma cell migration, invasion and EMT in vitro. Furthermore, mechanistic studies showed that matrix metallopeptidase-11 (MMP-11), was a direct target of miR-125a-5p in osteosarcoma. Taken together, the data demonstrate that miR-125a-5p functions as a tumor suppressor gene and serves an important role in inhibiting osteosarcoma cell migration, invasion and EMT by targeting MMP-11.
\end{abstract}

\section{Introduction}

Osteosarcoma is a primary malignant bone tumor with high morbidity in juveniles and adults, accounting for $\sim 60 \%$ of malignant bone tumors in the first two decades of life (1-3). Despite advances in diagnostic techniques and therapeutic management, the long-term survival of patients with osteosarcoma remains extremely low. The molecular mechanisms underlying the disease progression remain poorly understood $(4,5)$. Therefore, an improved understanding of the

Correspondence to: Professor Hong Yuan, Department of Orthopaedics, People's Hospital of Xinjiang Uygur Autonomous Region, 91 Tianchi Road, Urumqi, Xinjiang 830001, P.R. China E-mail: hongyuanuygur@126.com

${ }^{*}$ Contributed equally

Key words: osteosarcoma, miR-125a-5p, EMT, MMP-11 molecular mechanisms associated with the development and progression of human osteosarcoma is essential to find effective therapeutic interventions.

MicroRNAs (miRNAs) are small non-coding RNAs of 21-25 nucleotides in length that regulate the expression of target genes post-transcriptionally and serve important roles in various biological processes, including differentiation, development, proliferation and apoptosis (6-8). Emerging evidence shows that miRNAs are involved in the pathogenesis of the majority of types of cancer. The aberrant expression of miRNAs has been observed in a number of types of cancer, with this tightly associated with cell proliferation, migration, invasion and apoptosis. Previous studies have indicated that the expression of miR-125a-5p was significantly reduced in numerous types of tumors, including lung cancer (9), myeloma (10), colorectal adenomas and adenocarcinomas (11), and breast cancer (12). However, the biological role and the molecular mechanisms associated with miR-125a-5p in osteosarcoma remain unknown.

In the present study, the expression of miR-125a-5p was observed to be reduced in osteosarcoma tissues and cell lines compared with paired adjacent normal bone tissues and osteoblastic cells. The overexpression of miR-125a-5p inhibited cell migration, invasion and restrained epithelial-mesenchymal transition (EMT). In addition, at the molecular level, matrix metalloproteinase-11 (MMP-11) was a indicated to be a direct target of miR-125a-5p in osteosarcoma.

\section{Materials and methods}

Patient samples. A total of 28 paired osteosarcoma tissue specimens and adjacent matched normal osteosarcoma tissues were obtained from the People's Hospital of Xinjiang Uygur Autonomous Region. All samples were immediately snap-frozen in liquid nitrogen and stored at $-80^{\circ} \mathrm{C}$ until use. All patients (or patients' parents on behalf of the children) agreed to participate in the study and gave written informed consent. The study was approved by the Ethics Committee of People's Hospital of Xinjiang Uygur Autonomous Region.

Cell culture and transfection. Human osteosarcoma cell lines (HOS, Saos-2, MG-63 and U2OS) and the normal osteoblast cells were obtained from American Type Culture Collection (Manassas, VA, USA). All cells were cultured in Dulbecco's 
modified Eagle's medium (Invitrogen; Thermo Fisher Scientific, Inc., Waltham, MA, USA) supplemented with $10 \%$ fetal bovine serum (FBS; Invitrogen; Thermo Fisher Scientific, Inc.). Cultures were maintained at $37^{\circ} \mathrm{C}$ in a humidified atmosphere with $5 \% \mathrm{CO}_{2}$.

miR-125a-5p mimics and negative control were synthesized by Shanghai GenePharma Co., Ltd. (Shanghai, China). The sequences were as follows: Mimics, 5'-AGACCCUUU AACCUGUGAGGAC-3'; negative control, 5'-CAGUACUUU UGUGUAGUACAA-3'. A total of $5 \times 10^{6}$ MG-63 or U2OS cells were transfected with miR-125a-5p mimics or negative control using Lipofectamine 2000 (Invitrogen; Thermo Fisher Scientific, Inc.) following manufacturer's protocol.

Analysis of miR-125a-5p expression using reverse transcription-quantitative polymerase chain reaction ( $R T-q P C R)$. Total RNA from tissue samples and cell lines was harvested using TRIzol ${ }^{\circledR}$ reagent (Invitrogen; Thermo Fisher Scientific, Inc.). A total of $1 \mu \mathrm{g}$ of total RNA was reverse transcribed to cDNA using the miScript II RT kit (Qiagen GmbH, Hilden, Germany) according to the manufacturer's instruction. The RT reaction components were as follows: $5 \mathrm{X}$ miScript HiSpec Buffer $(4 \mu \mathrm{l})$, 10X Nucleics Mix ( $2 \mu \mathrm{l})$, miScript Reverse Transcriptase Mix $(2 \mu \mathrm{l})$, Template RNA (3 $\mu \mathrm{l})$ and RNase-free water $(9 \mu \mathrm{l})$. The RT protocol was follows: $60 \mathrm{~min}$ at $37^{\circ} \mathrm{C}, 5 \mathrm{~min}$ at $95^{\circ} \mathrm{C}$, then stored at $-20^{\circ} \mathrm{C}$. RT-qPCR was performed using an Applied Biosystems 7500 Real-time PCR System (Applied Biosystems; Thermo Fisher Scientific, Inc.) using the following cycling conditions: $95^{\circ} \mathrm{C}$ for $20 \mathrm{~min} ; 95^{\circ} \mathrm{C}$ for $10 \mathrm{sec}$; and $60^{\circ} \mathrm{C}$ for $20 \mathrm{sec}$ for 40 cycles. The relative expression ratio of miR-125a-5p in tissues and cells was quantified using the $2^{-\Delta \Delta C q}$ method (13). Small nuclear U6 snRNA was used as an internal control. The primers used for the amplification were as follows: miR-125a-5p forward primer, 5'-AGGACATCCAGGGTCACAGGTGA-3', reverse primer, miScript SYBR Green PCR kit Universal Primer; U6 forward primer, 5'-CTCGCTTCGGCAGCACA-3', reverse primer, 5'-ACGCTTCACGAATTTGCGT-3'.

Cell migration and invasion assays. A wound-healing assay was used to assess the effect of miR-125a-5p on cell migration ability in MG-63 and U2OS cells. An artificial wound was created $6 \mathrm{~h}$ following transfection using a pipette tip on the confluent cell monolayer, and to visualize migrated cells and wound healing, images were captured from five randomly selected fields in each sample taken at 0 and $12 \mathrm{~h}$. The effect of miR-125a-5p on cell invasion ability was examined using a Transwell assay. A total of $2 \times 10^{5}$ cells were plated in the top chamber of the insert pre-coated with Matrigel (BD Biosciences, Franklin Lakes, NJ, USA). Cells were plated in medium without serum, and medium supplemented with $10 \%$ FBS was added as a chemoattractant in the lower chamber. The cells were incubated for $48 \mathrm{~h}$, and the cells that migrated through the matrix to the other side of the insert were fixed with $100 \%$ methanol for $2 \mathrm{~min}$ at room temperature, stained with hematoxylin, and counted in 10 random fields per well (magnification, x100).

Bioinformatics. TargetScan (http://www.targetscan.org/), miRanda (http://www.targetscan.org/) and PicTar (http://pictar. mdc-berlin. de/) were used to predict the potential targets of miR-125a-5p in osteosarcoma.
A
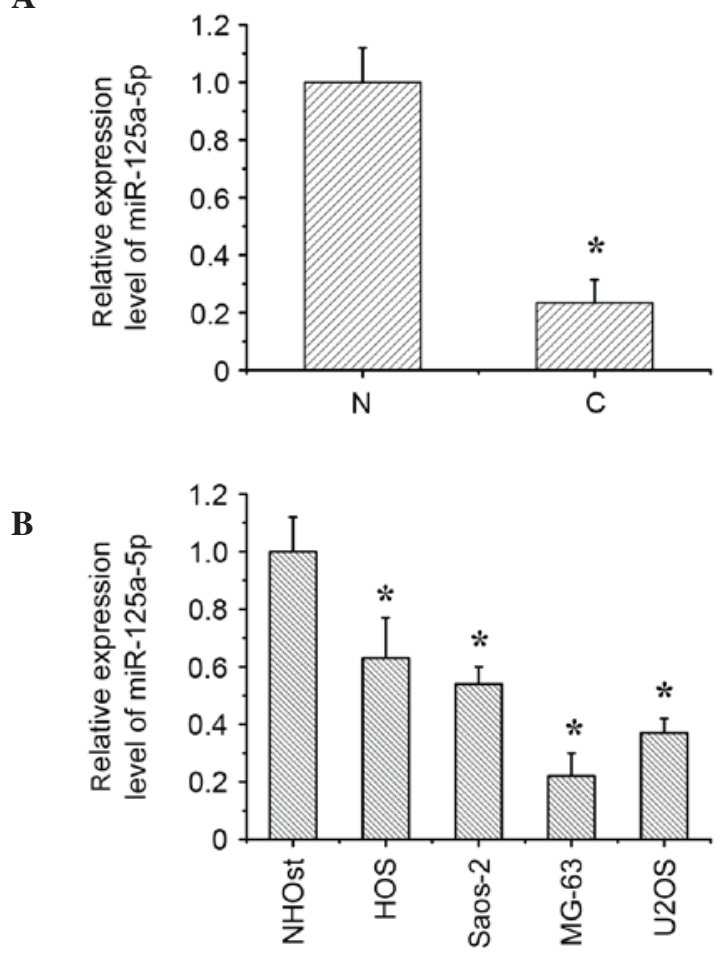

Figure 1. Expression levels of miR-125a-5p in osteosarcoma tissues and cell lines. (A) miR-125a-5p expression was determined by RT-qPCR in human osteosarcoma tissues and adjacent non-cancerous bone tissues. ${ }^{*} \mathrm{P}<0.05$ vs. $\mathrm{N}$ (B) RT-qPCR analysis of the expression levels of miR-125a-5p in osteosarcoma cell lines. Data are presented as the mean \pm standard deviation $(n=3)$. ${ }^{*} \mathrm{P}<0.05$ vs. NHOst. miR, microRNA; RT-qPCR, reverse transcription-quantitative polymerase chain reaction; $\mathrm{N}$, matched normal bone tissues; $\mathrm{C}$, osteosarcoma tissues; NHOst, normal osteoblast cells.

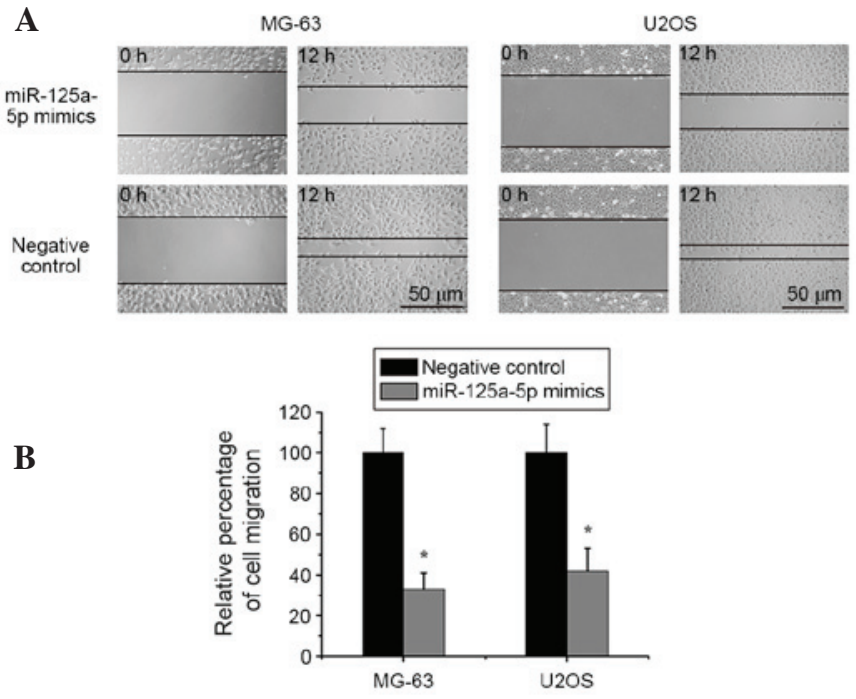

Figure 2. Restoration of miR-125a-5p expression inhibits cell migration in osteosarcoma. (A) A wound-healing assay was used to evaluate the migratory ability of MG-63 and U2OS cells following treatment with miR-125a-5p mimics or negative control. (B) The relative ratio of wound closure. Values are presented as the mean \pm standard deviaiton $(n=3)$. ${ }^{*} \mathrm{P}<0.01$ vs. the negative control. Scale bar $=50 \mu \mathrm{m} \cdot \mathrm{miR}$, microRNA.

Dual luciferase assays. Luciferase reporters were successfully constructed using molecular cloning technology. The target sequence was inserted into psiCHECK-2 
A

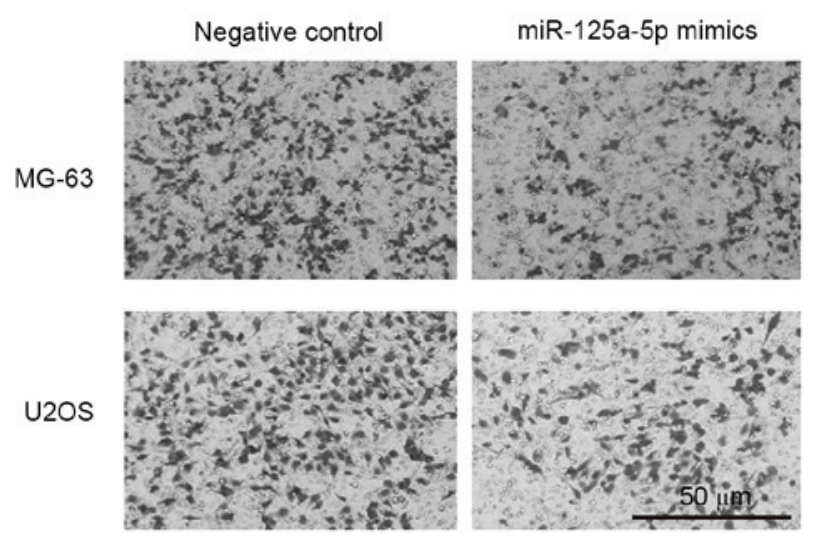

B

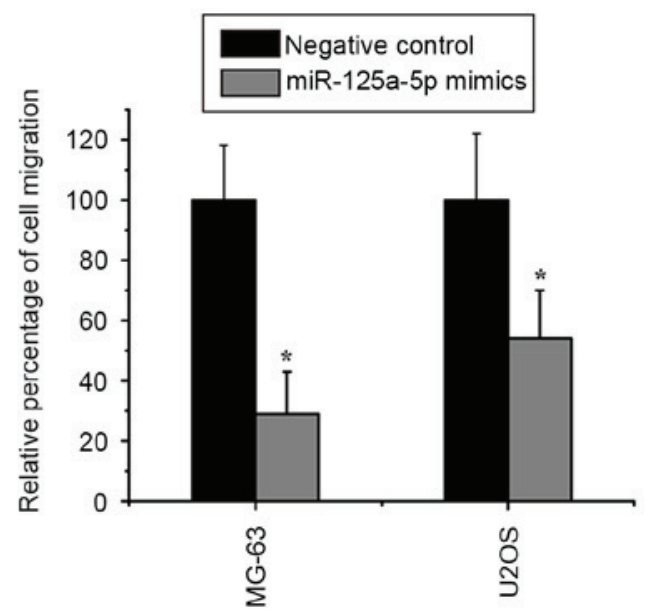

Figure 3. Restoration of miR-125a-5p expression inhibits cell invasion in osteosarcoma. (A) Transwell assay was used to evaluate MG-63 and U2OS cell invasion ability following transfection with miR-125a-5p mimics or negative control. (B) The relative ratio of invasive cells per field. Values are presented as the mean \pm standard deviation $(\mathrm{n}=3) .{ }^{*} \mathrm{P}<0.01$ vs. the negative control. Scale bar $=50 \mu \mathrm{m}$. miR, microRNA.

A



B

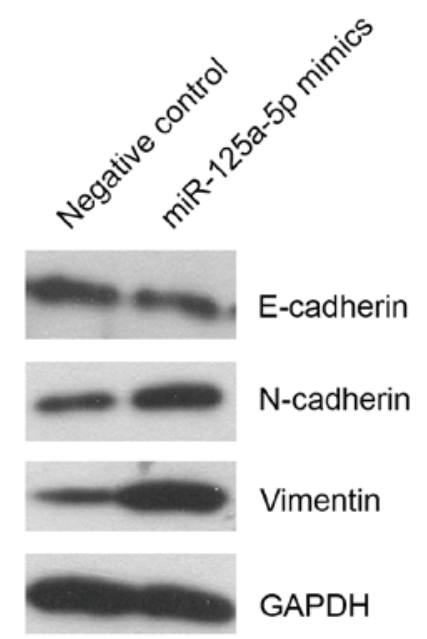

Figure 4. Restoration of miR-125a-5p expression reduced epithelial-mesenchymal transition in osteosarcoma. (A) Reverse transcription-quantitative polymerase chain reaction and (B) western blot analysis indicated that restorating the expression of miR-125a-5p reduced the expression levels of E-cadherin and increased the expression of $\mathrm{N}$-cadherin and vimentin in MG-63 cells. "P<0.01 vs. the negative control. miR, miRNA; GAPDH, glyceraldehyde 3-phosphate dehydrogenase.

luciferase reporter vectors (Promega Corporation, Madison, WI, USA) to obtain psiCHECK-2-MMP-11-WT-3'-untranslated region (UTR), which contains the miR-125a-5p binding sequence (MMP-11-3'-UTR sequence). The MMP-11 3'-UTRs contained sequences with mutations (psiCHECK-2- MMP-11-MUT-3'-UTR) in the putative binding site of MMP-11 3'-UTRs was chemically synthesized by Shanghai GenePharma Co., Ltd. MG-63 cells $\left(4 \times 10^{5}\right)$ were seeded in 24 -well plates and transfected with the psiCHECK-2-MMP-11-WT-3'-UTR or psiCHECK-2-MMP-11-MUT-3'-UTR recombinant plasmids using Lipofectamine 2000, and miR-125a-5p mimics or negative control for $24 \mathrm{~h}$. Luciferase values were determined using the Dual-Luciferase Reporter Assay System (Promega Corporation). The firefly luciferase gene was used as an internal control to normalize the transfection efficiency.
Western blotting. MG-63 cells were lysed with radioimmunoprecipitation assay lysis buffer (Invitrogen; Thermo Fisher Scientific, Inc.). The lysate was centrifuged at $13,000 \mathrm{x} \mathrm{g}$ for $20 \mathrm{~min}$ at $4^{\circ} \mathrm{C}$ and the supernatant was collected as total proteins. Protein concentration was determined using a bicinhoninic acid assay kit (Thermo Fisher Scientific, Inc.). Sodium dodecyl sulfate polyacrylamide gel electrophoresis (12\%; Invitrogen; Thermo Fisher Scientific, Inc.) was used to separate the proteins $(40 \mu \mathrm{g})$ prior to transfer to polyvinylidene fluoride membranes (EMD Millipore, Billerica, MA, USA). Following blocking with $5 \%$ non-fat milk for $2 \mathrm{~h}$, membranes were incubated with the following primary antibodies overnight at $4{ }^{\circ} \mathrm{C}$ : Mouse monoclonal anti-E-cadherin (1:500; sc-8426), mouse monoclonal anti-N-cadherin (1:1,000; sc-271386), mouse monoclonal anti-vimentin (1:500; sc-373717), rabbit polyclonal anti-MMP-11 (1:200; sc-8836-R), and mouse monoclonal 
A

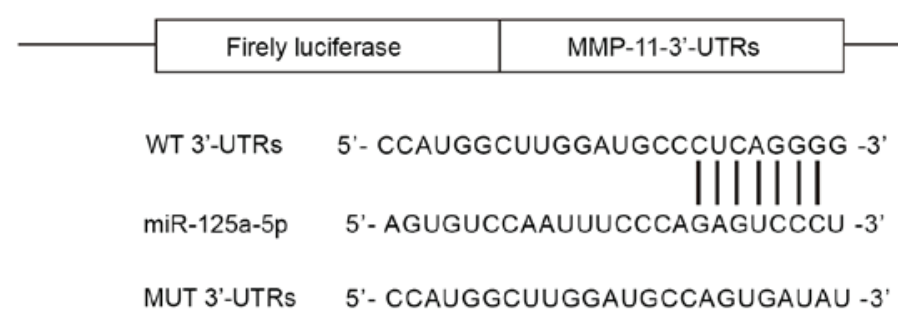

B

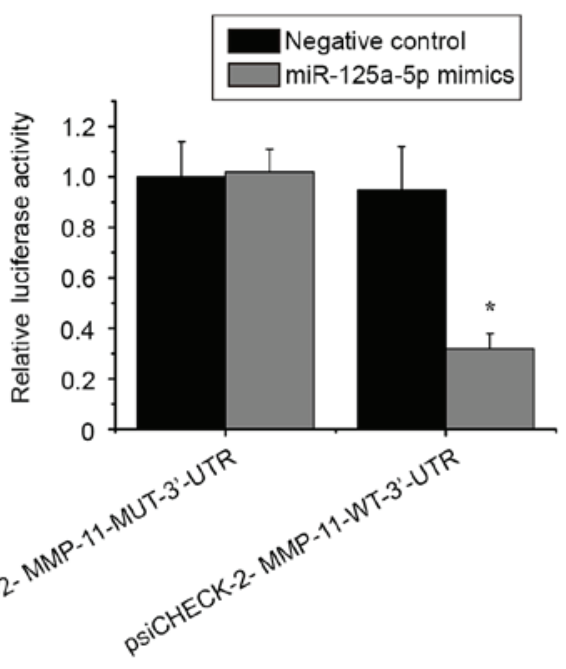

C
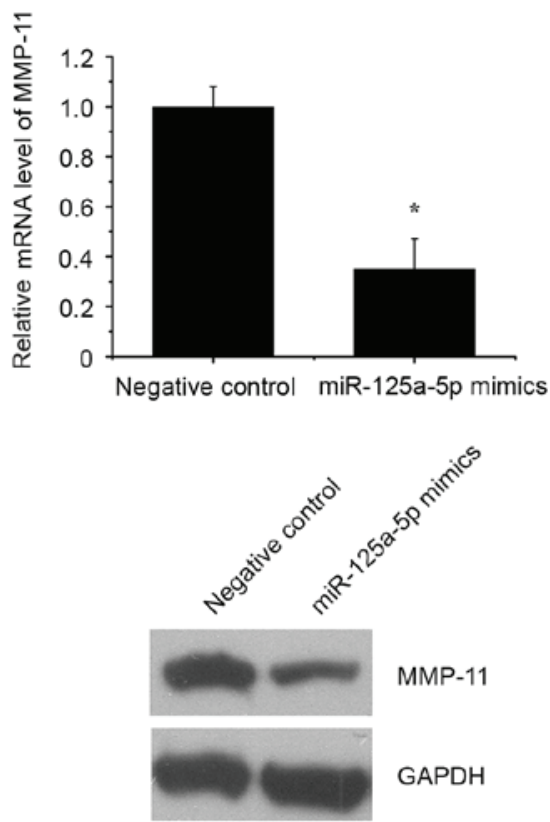

Figure 5. MMP-11 is a direct target of miR-125a-5p in osteosarcoma cells. (A) The sequences of the miR-125a-5p binding sites within the human MMP-11 mRNA 3'UTR and a schematic of the reporter constructs. The psiCHECK-2-MMP-11-WT-3'-UTR was the recombinant reporter plasmid containing the entire 3'UTR sequences of MMP-11, and psiCHECK-2-MMP-11-MUT-3'-UTR was the reporter construct containing the mutated nucleotides. (B) The relative luciferase activitie of the psiCHECK-2- MMP-11-WT-3'-UTR and psiCHECK-2-MMP-11-WT-3'-UTR in MG-63 cells. (C) Overexpression of miR-125a-5p reduced the mRNA and protein expression levels of MMP-11 in MG-63 cells measured by reverse transcription-quantitative polymerase chain reaction and western blotting. "P<0.001. MMP, matrix metalloproteinase; miR, microRNA; UTR, untranslated region; WT, wild type; MUT, mutant; GAPDH, glyceraldehyde 3-phosphate dehydrogenase.

anti-GAPDH antibody (1:2,000; sc-365062; all from Santa Cruz Biotechnology, Inc., Dallas, TX, USA). Subsequently, membranes were incubated for $1 \mathrm{~h}$ at room temperature with rabbit anti-mouse IgG (1:5,000 ab6728; Abcam, Cambridge, UK) and goat anti-mouse IgG (1:5,000; ab97051; Abcam). The protein bands detected by enhanced chemiluminescence (GE Healthcare Life Sciences, Little Chalfont, UK). The levels of GAPDH were used as loading controls. Western blot bands were obtained using Imaging System, and the protein density was quantified using Odyssey software, version 1.2 (LI-COR Biosciences, Lincoln, NE, USA).

Statistical analysis. Statistical analysis was performed using SPSS software, version 17.0 (SPSS, Inc., Chicago, IL, USA) and data are presented as the mean \pm standard error. The miR-125a-5p expression differences between osteosarcoma tissues and normal bone tissues were analyzed using a paired samples t-test. The miR-125a-5p expression in osteosarcoma cell lines was analysed using one-way analysis of variance and dual luciferase assay was analyzed using two-way analysis of variance and Dunnett's test. The other data was analyzed using an independent-samples t-test. $\mathrm{P}<0.05$ was considered to indicate a statistically significant difference. All experiments were repeated a minimum of three times.

\section{Results}

The expression of miR-125a-5p is downregulated in osteosarcoma tissues and cell lines. The expression levels of miR-125a-5p were downregulated in osteosarcoma tissues compared with the matched normal bone tissues, as measured by RT-qPCR (Fig. 1A). The miR-125a-5p expression levels in osteosarcoma cell lines (HOS, Saos-2, MG-63, and U2OS) were additionally downregulated compared with the normal osteoblast cells (Fig. 1B).

Restoration of miR-125a-5p expression inhibits cell migration and invasion in osteosarcoma. In order to assess the effects of miR-125a-5p on osteosarcoma cell migration and invasion, miR-125a-5p mimics or negative controls were transfected into MG-63 and U2OS cells. Wound-healing and Transwell assays indicated that enhancing the expression levels of miR-125a-5p markedly inhibited migration and invasion compared with the control group in MG-63 and U2OS cells (Figs. 2 and 3). 
Restoration of miR-125a-5p expression reduced EMT in osteosarcoma. As indicated by RT-qPCR and western blotting, the overexpression of miR-125a-5p in MG-63 cells reduced the expression of E-cadherin and increased the expression of N-cadherin and vimentin (Fig. 4), which suggested that miR-125a-5p inhibits EMT in osteosarcoma.

MMP-11 is a direct target of miR-125a-5p in osteosarcoma. To explore the downstream targets of miR-125a-5p, bioinformatics was performed to identify potential targets. MMP-11 was identified as a putative gene by the bioinformatics prediction, with its 3'-UTR containing a complementary site for the seed region of miR-125a-5p (Fig. 5A).

In order to obtain further direct evidence that MMP-11 was a target of miR-125a-5p, the binding site of miR-125a-5p in the 3'UTR of MMP-11 mRNA was characterized. The results indicated that miR-125a-5p significantly reduced the luciferase activity, while the mutant reporter co-transfected with miR-125a-5p did not result in a significant reduction in the relative luciferase activity (Fig. 5B). Subsequently, RT-qPCR and western blot analysis was conducted to examine the inhibitory effect of miR-125a-5p on endogenous MMP-11 expression in MG-63 cells. As expected, inducing miR-125a-5p expression markedly inhibited the expression of MMP-11 in MG-63 cells (Fig. 5C).

\section{Discussion}

Increasingly, studies have demonstrated that miR-125a-5p is frequently abnormally expressed in a variety of human malignancies, including glioblastoma (14), gastric cancer (15), non-small cell lung cancer (16), oral squamous cell carcinoma (17) and hepatocellular carcinoma (18). Downregulation of miR-125a-5p inhibits the cell cycle, proliferation and induces apoptosis by directly targeting the ErbB pathway in acute myeloid leukemia (19). miRNA-125a-5p inhibits glioblastoma cell proliferation and promotes cell differentiation by targeting transcriptional coactivator with PDZ-binding motif (20). In addition, miR-125a-5p has been demonstrated to suppress breast tumorigenesis by targeting histone deacetylase 4 (21). In the present study, miR-125a-5p was observed to be downregulated in osteosarcoma tissues and cell lines. Additionally, restoration of miR-125a-5p expression resulted in reductions in osteosarcoma cell migration, invasion and the inhibition of cellular EMT. These results suggest that miR-125a-5p acts as a tumor suppressor gene, and may contribute to the development and metastasis of osteosarcoma.

It has been previously reported that MMPs contribute to cell migration and invasion in various types of cancer (22). MMPs are a family of zinc-dependent extracellular endoproteinases, which have the ability to degrade the cell extracellular matrix and basement membrane (23). To date, greater than 24 MMPs have been identified in humans, and are classified into five groups based on substrate specificity: Interstitial collagenases, gelatinases, stromelysins, matrilysins and membrane-type MMPs (24,25). MMP-11, also termed stromelysin-3, is encoded by the MPP-11 gene located on chromosome 22 at q11.23 in the human genome (26). Increasingly, studies have demonstrated that MMP-11 is frequently highly expressed in numerous types of cancer, including breast, brain, gastric and lung cancer (27). A previous study reported that MMP-11 deficiency resulted in an increased tumor-free survival rate and modulated the distant invasion ability of tumors (28). The results from the present study suggest that MMP-11 is an important downstream target of miR-125a-5p in osteosarcoma. miR-125a-5p directly binds to the 3'-UTR of MMP-11, which was verified by the dual-luciferase reporter assay. In addition, upregulation of miR-125a-5p markedly reduced the MMP-11 protein expression levels in osteosarcoma cells. Together, these data suggest that miR-125a-5p suppresses migration, invasion and EMT through direct targeting of MMP-11 in osteosarcoma.

In conclusion, the present study demonstrated that miR-125a-5p was downregulated in osteosarcoma tissues and cell lines. Restoration of miR-125a-5p expression inhibited cell migration, invasion and EMT of osteosarcoma through the direct targeting of MMP-11. To the best of our knowledge, the present study is the first to demonstrate that the miR-125a-5p/MMP-11 axis regulates the migration, invasion and EMT of osteosarcoma cells. These observations indicate that miR-125a-5p may be a potential novel target for gene therapy of osteosarcoma.

\section{References}

1. Tang J, Shen L, Yang Q and Zhang C: Overexpression of metadherin mediates metastasis of osteosarcoma by regulating epithelial-mesenchymal transition. Cell Prolif 47: 427-434, 2014.

2. Montanaro L, Mazzini G, Barbieri S, Vici M, Nardi-Pantoli A, Govoni M, Donati G, Treré D and Derenzini M: Different effects of ribosome biogenesis inhibition on cell proliferation in retinoblastoma protein- and p53-deficient and proficient human osteosarcoma cell lines. Cell Prolif 40: 532-549, 2007.

3. Li G, Cai M, Fu D, Chen K, Sun M, Cai Z and Cheng B: Heat shock protein $90 \mathrm{~B} 1$ plays an oncogenic role and is a target of microRNA-223 in human osteosarcoma. Cell Physiol Biochem 30: 1481-1490, 2012.

4. McQueen P, Ghaffar S, Guo Y, Rubin EM, Zi X and Hoang BH: The Wnt signaling pathway: Implications for therapy in osteosarcoma. Expert Rev Anticancer Ther 11: 1223-1232, 2011.

5. Wu CL, Tsai HC, Chen ZW, Wu CM, Li TM, Fong YC and Tang CH: Ras activation mediates WISP-1-induced increases in cell motility and matrix metalloproteinase expression in human osteosarcoma. Cell Signal 25: 2812-2822, 2013.

6. Bonazzi VF, Stark MS and Hayward NK: MicroRNA regulation of melanoma progression. Melanoma Res 22: 101-113, 2012.

7. Martello G, Rosato A, Ferrari F, Manfrin A, Cordenonsi M, Dupont S, Enzo E, Guzzardo V, Rondina M, Spruce T, et al: A MicroRNA targeting dicer for metastasis control. Cell 141: 1195-1207, 2010

8. Mo YY: MicroRNA regulatory networks and human disease. Cell Mol Life Sci 69: 3529-3531, 2012.

9. Jiang L, Huang Q, Chang J, Wang E and Qiu X: MicroRNA HSA-miR-125a-5p induces apoptosis by activating p53 in lung cancer cells. Exp Lung Res 37: 387-398, 2011.

10. Leotta M, Biamonte L, Raimondi L, Ronchetti D, Di Martino MT, Botta C, Leone E, Pitari MR, Neri A, Giordano A, et al: A p53-dependent tumor suppressor network is induced by selective miR-125a-5p inhibition in multiple myeloma cells. J Cell Physiol 229: 2106-2116, 2014.

11. Gattolliat CH, Uguen A, Pesson M, Trillet K, Simon B, Doucet L, Robaszkiewicz M and Corcos L: MicroRNA and targeted mRNA expression profiling analysis in human colorectal adenomas and adenocarcinomas. Eur J Cancer 51: 409-420, 2015.

12. Hsieh TH, Hsu CY, Tsai CF, Long CY, Wu CH, Wu DC, Lee JN, Chang WC and Tsai EM: HDAC inhibitors target HDAC5, upregulate microRNA-125a-5p, and induce apoptosis in breast cancer cells. Mol Ther 23: 656-666, 2015.

13. Schmittgen TD and Livak KJ: Analyzing real-time PCR data by the comparative C(T) method. Nat Protoc 3: 1101-1108, 2008 
14. Cortez MA, Nicoloso MS, Shimizu M, Rossi S, Gopisetty G, Molina JR, Carlotti C Jr, Tirapelli D, Neder L, Brassesco MS, et al: $\mathrm{miR}-29 \mathrm{~b}$ and $\mathrm{miR}-125 \mathrm{a}$ regulate podoplanin and suppress invasion in glioblastoma. Genes Chromosomes Cancer 49: 981-990, 2010

15. Hashiguchi Y, Nishida N, Mimori K, Sudo T, Tanaka F, Shibata K, Ishii H, Mochizuki H, Hase K, Doki Y and Mori M: Down-regulation of miR-125a-3p in human gastric cancer and its clinicopathological significance. Int J Oncol 40: 1477-1482, 2012.

16. Jiang L, Huang Q, Zhang S, Zhang Q, Chang J, Qiu X and Wang E: Hsa-miR-125a-3p and hsa-miR-125a-5p are downregulated in non-small cell lung cancer and have inverse effects on invasion and migration of lung cancer cells. BMC Cancer 10: 318, 2010.

17. Tiwari A, Shivananda S, Gopinath KS and Kumar A MicroRNA-125a reduces proliferation and invasion of oral squamous cell carcinoma cells by targeting estrogen-related receptor $\alpha$ : Implications for cancer therapeutics. J Biol Chem 289: 32276-32290, 2014.

18. Kim JK, Noh JH, Jung KH, Eun JW, Bae HJ, Kim MG, Chang YG, Shen Q, Park WS, Lee JY, et al: Sirtuin7 oncogenic potential in human hepatocellular carcinoma and its regulation by the tumor suppressors MiR-125a-5p and MiR-125b. Hepatology 57: 1055-1067, 2013

19. Ufkin ML, Peterson S, Yang X, Driscoll H, Duarte C and Sathyanarayana P: miR-125a regulates cell cycle, proliferation and apoptosis by targeting the ErbB pathway in acute myeloid leukemia. Leuk Res 38: 402-410, 2014.

20. Yuan J, Xiao G, Peng G, Liu D, Wang Z, Liao Y, Liu Q, Wu M and Yuan X: MiRNA-125a-5p inhibits glioblastoma cell proliferation and promotes cell differentiation by targeting TAZ. Biochem Biophys Res Commun 457: 171-176, 2015.
21. Hsieh TH, Hsu CY, Tsai CF, Long CY, Chai CY, Hou MF, Lee JN, Wu DC, Wang SC and Tsai EM: miR-125a-5p is a prognostic biomarker that targets HDAC4 to suppress breast tumorigenesis. Oncotarget 6: 494-509, 2015.

22. King SE: Matrix metalloproteinases: New directions toward inhibition in the fight against cancers. Future Med Chem 8: 297-309, 2016.

23. Yan D, Dai H and Liu JW: Serum levels of MMP-11 correlate with clinical outcome in Chinese patients with advanced gastric adenocarcinoma. BMC Cancer 11: 151, 2011.

24. Kähäri VM and Saarialho-Kere U: Matrix metalloproteinases and their inhibitors in tumour growth and invasion. Ann Med 31: 34-45, 1999.

25. Clark AF: New discoveries on the roles of matrix metalloproteinases in ocular cell biology and pathology. Invest Ophthalmol Vis Sci 39: 2514-2516, 1998.

26. Valdivia A, Peralta R, Matute-González M, García Cebada JM, Casasola I, Jiménez-Medrano C, Aguado-Pérez R, Villegas V, González-Bonilla C, Manuel-Apolinar L, et al: Co-expression of metalloproteinases 11 and 12 in cervical scrapes cells from cervical precursor lesions. Int J Clin Exp Pathol 4: 674-682, 2011.

27. Wu D, Li M, Wang L, Zhou Y, Zhou J, Pan $\mathrm{H}$ and Qu P. microRNA145 inhibits cell proliferation, migration and invasion by targeting matrix metallopeptidase-11 in renal cell carcinoma. Mol Med Rep 10: 393-398, 2014.

28. Peruzzi D, Mori F, Conforti A, Lazzaro D, De Rinaldis E, Ciliberto G, La Monica N and Aurisicchio L: MMP11: A novel target antigen for cancer immunotherapy. Clin Cancer Res 15: 4104-4113, 2009. 